\title{
CCD PHOTOMETRY OF THE NUCLEI OF THREE SUPERGIANT ELLIPTICAL GALAXIES: EVIDENCE FOR A SUPERMASSIVE OBJECT IN THE CENTER OF THE RADIO GALAXY NGC 6251
}

\author{
Peter J Young, ${ }^{1,2}$ Wallace L. W. Sargent, ${ }^{1,2}{ }^{\text {Jerome Kristian, }}{ }^{3}$ AND \\ JAMES A. WESTPHAL ${ }^{1}$ \\ Received 1979 April 5; accepted 1979 June 5
}

\begin{abstract}
We have analyzed charged coupled device (CCD) photometry in the $g$ and $r$ bands of three supergiant elliptical galaxies: NGC 4874 and NGC 4889 in the Coma cluster, and the radio galaxy NGC 6251. The profiles of NGC 4874 and NGC 4889 are closely fitted by King models. The profile of NGC 6251 cannot be fitted by a King model, nor by a King model plus a central point luminosity source (the presence of which is suggested by a blue trend in the colors at small radii). The previously studied profile of $\mathrm{M} 87$, when convolved with an appropriate Gaussian function to allow for the difference in distance, closely resembles the profile of NGC 6251 (but not those of NGC 4874 or NGC 4889). NGC 6251 was successfully fitted with a luminosity profile containing both a point luminosity source and a central point mass. The value of this point mass cannot be determined without additional dynamical data. However, if the core velocity dispersion is similar to that of other giant ellipticals of the same absolute magnitude, the point mass is likely to be about $2.4 \times 10^{9} M_{\odot}$, similar to the value reported earlier for M87.

The data are also used to derive upper limits for possible central point masses in NGC 4874 and NGC 4889.
\end{abstract}

Subject headings: galaxies: individual — galaxies: nuclei - galaxies: photometry galaxies: structure

\section{INTRODUCTION}

In two previous papers (Young et al. 1978; Sargent et al. 1978), we presented evidence that the supergiant elliptical galaxy M87 (NGC 4486) contains a large central mass which is superposed on a mass distribution which can otherwise be represented very adequately by a King model. In essence, we showed that the observations demand a mass of $3 \times 10^{9} M_{\odot}$ inside a radius of $110 \mathrm{pc}$ with a mass-to-light ratio $M / L=60$; this is to be compared with a value $M / L=6$ for the whole of the rest of the galaxy out to a radius of $10 \mathrm{kpc}$. We suggested that the most plausible explanation of our results was that M87 contains a central black hole of $3 \times 10^{9} M_{\odot}$. However, on the basis of the present observations it is not possible to exclude other possibilities-for example, that M87 contains a dynamically distinct core in which the stellar population is very different from that in the rest of the galaxy. The main evidence against such a suggestion is that the line strength index measured in the spectroscopic work

${ }^{1}$ Hale Observatories, California Institute of Technology. The Hale Observatories is operated jointly by the Carnegie Institution of Washington and the California Institute of Technology.

2 Visiting Astronomer, Kitt Peak National Observatory, which is operated by Associated Universities for Research in Astronomy, Inc., under contract from the National Science Foundation.

${ }^{3}$ Hale Observatories, Carnegie Institution of Washington. The Hale Observatories is operated jointly by the Carnegie Institution of Washington and the California Institute of Technology. of Sargent et al. shows no perceptible variation in the immediate vicinity of the center of M87; consequently, we have no evidence at present that the stellar population in the core of M87 differs in any way from that at larger radii.

The existence of a central mass in M87 was deduced from accurate measurements of the light distribution and of the velocity dispersion as a function of radius. The photometric results have since been confirmed by de Vaucouleurs and Nieto (1979), while the velocity dispersion measurements for the critical inner part of M87 have been confirmed by Rose and Searle (1979, private communication). Therefore, since the anomalies in the light profile of M87 are so large and easily detectable, we have been encouraged to extend our observations to more distant supergiant elliptical galaxies. This paper presents the results of CCD photometry of the central giant galaxies NGC 4874 and NGC 4889, two giant galaxies in the center of the Coma cluster, and of the extraordinary radio galaxy NGC 6251.

The newly discovered radio source associated with the elliptical galaxy NGC 6251 has an angular size of $1: 2$ corresponding to a projected size of $3.0 \mathrm{Mpc}$. A prominent feature of the radio source is a remarkable jet which is straight for nearly all of its $200 \mathrm{kpc}$ projected length (Waggett, Warner, and Baldwin 1977). VLBI observations by Readhead, Cohen, and Blandford (1978) showed that the core of the radio source, which is coincident with the center of NGC 6251, contains a much smaller jet with a projected length of 
$1.7 \mathrm{pc}$ : the position angles of the inner and outer jets are identical within the errors of measurement; moreover, they point roughly along the minor axis of the galaxy. Recent observations by Readhead, Sargent, and de Bruyn (1979) have shown that NGC 6251 lies on the edge of a loose cluster of galaxies $\mathrm{Zw} \mathrm{Cl}$ $1609.0+8212$ which was delineated by Zwicky and Herzog (1968). The redshift of the NGC 6251 cluster is $c z=7110 \mathrm{~km} \mathrm{~s}^{-1}$, quite close to that of the Coma cluster $\left(c z=6645 \mathrm{~km} \mathrm{~s}^{-1}\right)$. NGC 6251 thus lies at a distance of $119 \mathrm{Mpc}$ (assuming $H_{0}=60 \mathrm{~km} \mathrm{~s}^{-1}$ $\mathrm{Mpc}^{-1}$ ); its absolute magnitude is $M_{B(0)}=-21.3$. The spectrum of NGC 6251 is that of a normal giant elliptical galaxy except that there is a weak, sharp emission line due to [O III] $\lambda 5007$. In this respect NGC 6251 resembles M87 which also has weak emission lines from the central core (Arp 1967; Boksenberg, Sargent, and Young, unpublished).

Of the two Coma galaxies, NGC 4874 is associated with the weak radio source 5C 4.85 (Willson 1970); this galaxy is unique among the Coma cluster ellipticals in having weak [O II] $\lambda 3727$ emission in its optical spectrum. NGC 4889 is not a known radio source.

In the rest of this paper, the observations of the three galaxies are described in $\S \mathrm{II}$, while the derivation of the radial light profiles is described in $\S$ III. In $\$$ IV we show that the light profiles of NGC 4874 and NGC 4889 conform, to very high precision, to the predictions of suitable King models. On the other hand, the light profile in the center of NGC 6251 shows clear departures from King model predictions. We show that when the appropriate allowance is made for the difference in distance, the central light profiles in M87 and NGC 6251 are very similar. A discussion of the consequences of this discovery is contained in $\S \mathrm{V}$.

\section{OBSERVATIONS AND REDUCTIONS}

The observations of NGC 4874 and NGC 4889, and most of the observations of NGC 6251, were made at the prime focus of the Kitt Peak 4 m telescope in 1978 May during a dark run which was devoted to trials of a CCD camera built by the Jet Propulsion Laboratory. The actual CCD chip used was the same $400 \times 400$ array of $25 \mu$ square pixels as was used in the Palomar observations of M87 which were reported by Young et al. (1978). However, the associated electronics were supplied by JPL. The image scale at Kitt Peak was 0 ".446 per pixel, giving a $3^{\prime}$ field with the $\mathrm{f} / 2.9$ beam.

In order to optimize the observations with due regard for the spectral response of the CCD, we made observations through Gunn's $g$ or $r$ filters (Thuan and Gunn 1976). The $r$ filter was combined with an additional $\mathrm{KGl}$ component in order to block the near-infrared response of the CCD and hence cut down difficulties with interference fringes produced in the chip.

The Palomar observations were made in exactly the same manner as those described by Young et al. (1978).

A journal of observations listing the exposures on the three galaxies is given in Table 1. The sky levels were determined by making similar exposures on suitable blank fields which were typically about $30^{\prime}$ away from the galaxies. Six standard stars from the list published by Thuan and Gunn (1976) were observed in order to provide absolute calibration of the galaxy photometry.

The initial reductions were performed according to the following procedure. In CCD photometry four types of data frames are obtained:

1. Dark-count frames in which the CCD is exposed to total darkness for periods $t_{D}$ which range in practice from 10 to 60 minutes. Generally, the shorter dark exposures were used in the reductions because they had fewer cosmic ray events. Let the dark signal from pixel $(i, j)$ be $D_{i j}$.

2. Galaxy exposures $G_{i j}$ with exposure time $t_{G}$ seconds.

3. DC-offset "bias" frames $B_{i j}$ obtained by exposing the CCD to total darkness for a brief period ( $\sim 0.1 \mathrm{~s})$ and then reading it out.

4. Flat field frames $F_{i j}$. These are short exposures made on a portion of the dome which was illuminated by a current-regulated lamp. The flat field exposures at Kitt Peak were made with a binary power series of exposure times ( $1 \mathrm{~s}, 2 \mathrm{~s}, 4 \mathrm{~s}$, etc.). It was found from these exposures that there were significant $(\sim 5 \%)$ nonlinearities in the response of the system. The nonlinearities were found to be stable and to be well

TABLE 1

JOURNAL OF OBSERVATIONS

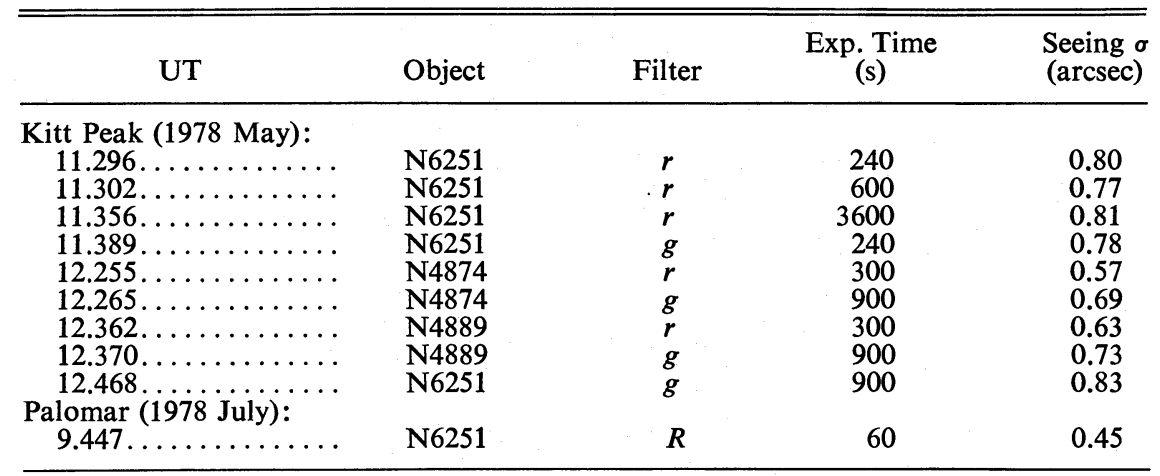


represented by a single transfer function 2. This function was determined from the binary series of flat field exposures by Lynds (1978, private communication); when applied to the flat field data, the initial nonlinearities were removed to better than $0.5 \%$.

The reduced data were then obtained for each galaxy exposure from the equation

$$
g_{i j}=\frac{\mathscr{2}\left[G_{i j}-B_{i j}\right]-\left(t_{G} / t_{D}\right) \mathscr{2}\left[D_{i j}-B_{i j}\right]}{2\left[F_{i j}-B_{i j}\right]} .
$$

The Palomar observations were not subject to the nonlinearities found at Kitt Peak. In this case the transfer function $\mathscr{Q}$ was set equal to unity.

In the reductions of the Gunn $g, r$ standards we used new magnitude and color determinations by Kent (1979, private communication). Mean extinction coefficients for Palomar (scaled to the altitude of Kitt Peak) were used. After determining the photometric transformations of the CCD, the standard stars left residuals of $\sim 0.02 \mathrm{mag}$. Series of exposures on an object with the CCD through the course of the two nights showed that the sky transmission was stable to $\sim 3 \%$.

\section{GALAXY PROFILES}

\section{a) Ellipticities}

The ellipticities of the galaxies as a function of radius were determined by the following procedure. Consider Cartesian $(x, y)$ and $(\tilde{x}, \tilde{y})$ coordinates such that

$$
\begin{aligned}
& \tilde{x}=x_{c}+x \cos \psi+y \sin \psi, \\
& \tilde{y}=y_{c}-x \sin \psi+y \cos \psi .
\end{aligned}
$$

Suppose the galaxy profile to be of the form

$$
\begin{aligned}
I & =I(s), \\
s^{2} & =c \tilde{x}^{2}+c^{-1} \tilde{y}^{2} .
\end{aligned}
$$

Consider data in a narrow elliptical annulus traced out by

$$
x=\alpha \cos \theta ; \quad y=\beta \sin \theta ; \quad \theta \in[0,2 \pi),
$$

which is given by

$$
I=I\left(s_{0}\right)+\left(s-s_{0}\right) I^{\prime}\left(s_{0}\right),
$$

with $s$ supplied by equations (2), (3b), and (4). In considering $I$ as a function of $\theta$ we may express it in the form

$$
\begin{aligned}
I=I_{0} & +A_{1} \cos \theta+B_{1} \sin \theta \\
& +A_{2} \cos 2 \theta+B_{2} \sin 2 \theta,
\end{aligned}
$$

with

$$
\begin{aligned}
& A_{1}=\frac{\alpha I^{\prime}}{(\alpha \beta)^{1 / 2}}\left[c x_{c} \cos \psi-\left(y_{c} / c\right) \sin \psi\right], \\
& B_{1}=\frac{\beta I^{\prime}}{(\alpha \beta)^{1 / 2}}\left[c x_{c} \sin \psi+\left(y_{c} / c\right) \cos \psi\right],
\end{aligned}
$$

$$
\begin{gathered}
A_{2}=\frac{1}{8}(\alpha \beta)^{1 / 2} I^{\prime}\left[\left(c+c^{-1}\right)\left(\gamma^{-1}-\gamma\right)+\left(c-c^{-1}\right)\right. \\
\left.\quad \times\left(\gamma+\gamma^{-1}\right) \cos 2 \psi\right], \\
B_{2}=\frac{1}{4}(\alpha \beta)^{1 / 2} I^{\prime}\left(c-c^{-1}\right) \sin 2 \psi,
\end{gathered}
$$

where $I^{\prime}=I^{\prime}\left(s_{0}\right) ; s_{0}=(\alpha \beta)^{1 / 2} ; \gamma=\beta / \alpha$.

To analyze the data, the Fourier components are derived from a narrow elliptical annulus. If any of the quantities $x_{c}, y_{c}, \psi,(\gamma-c)$ are nonzero, then the annulus does not scan around an isophote of the galaxy, and the Fourier harmonics are nonzero. To derive the true ellipticity from the quantities $A_{2}, B_{2}$, we solve:

$$
\begin{aligned}
K= & {\left[\left(\gamma+\gamma^{-1}\right)^{2}+4{a_{2}}^{2}+b_{2}{ }^{2}\left(\gamma+\gamma^{-1}\right)^{2}\right.} \\
& \left.+a_{2}{ }^{2}\left(\gamma-\gamma^{-1}\right)^{2}\right]^{1 / 2}+a_{2}\left(\gamma-\gamma^{-1}\right), \\
c= & {\left[K-\left(K^{2}-4\right)^{1 / 2}\right]^{1 / 2}, }
\end{aligned}
$$

for the quantity $c=b / a$, the axis ratio of the galaxy. Here $a_{2}=2 A_{2} /(\alpha \beta)^{1 / 2} I^{\prime} ; b_{2}=2 B_{2} /(\alpha \beta)^{1 / 2} I^{\prime}$. The pair of equations (6d), (6e) yields a quartic in $c$ (note that if $c, \psi$ satisfy equations [6d], [6e] then $c^{-1}, \psi+\frac{1}{2} \pi$ do also), and equation (7) gives the solitary root with $0<c \leq 1$. The rest of equation (6) then immediately gives the angle correction $\psi$ and the centroid offset $\left(x_{c}, y_{c}\right)$.

\section{b) Profiles}

In order to determine the galaxy profiles, we first choose a mean axis ratio $c=b / a$ and position angle $\psi$. With the radial parameter $s$ as defined in equations (2) and (3), all pixels with $s_{\min }<s \leq s_{\max }$ are selected from the data to define the mean count level in a particular elliptic annulus, the center of a pixel being used to define $s$. Near the center of the galaxy, where the discrete sampling of the data becomes important, a two-dimensional quadratic polynomial is splined to $3 \times 3$ blocks of pixels. With the polynomial the central pixel of the $3 \times 3$ block is broken up into $n^{2}$ subpixels (the closer to the center of the galaxy, the more subpixels are used). The subpixels are then cast into the bins in the coordinate $s$.

We then have the galaxy profile $I(s)$ integrated in elliptical annuli. The intervals chosen have $\Delta \sinh ^{-1}\left(s / s_{*}\right)=0.07$, with $s_{*}=1$ pixel. The function $I(s)$ is approximated locally with cubic polynomials, integrated over the annuli, and spline fitted to the data points. The use of cubic spline functions has the advantage that the first derivative of $I(s)$, which is required in the ellipticity determination, is continuous at the boundaries of the annuli. At the same time the well-known disadvantages associated with fitting higher order polynomials to empirical data are avoided.

Since the sky exposures showed fluctuations at the $1-2 \%$ level we truncated our photometric profiles at $s=17^{\prime \prime}$ in order that possible systematic errors from inaccurate sky subtraction be less than $0.02 \mathrm{mag}$.

\section{c) Results}

In Tables 2-4 we give the mean profiles and ellipticities as determined by the methods of $\S \mathrm{III} a, b$. In 
TABLE 2

N4874 PHOTOMETRY

\begin{tabular}{|c|c|c|c|c|c|c|}
\hline $\begin{array}{c}\text { Radius } \\
\text { (Arcsec) }\end{array}$ & $\mu_{r}^{a}$ & $g-r^{a}$ & $\begin{array}{c}\varepsilon^{b} \\
(1-b / a)\end{array}$ & $\dot{\theta}^{\mathrm{P} \cdot \mathrm{A}}{ }^{\mathrm{b}}$ & $\begin{array}{c}\Delta \mathrm{x}^{\prime \prime} \mathrm{b} \\
(\text { North) }\end{array}$ & $\begin{array}{c}\Delta y^{\prime \prime b} \\
\text { (East) }\end{array}$ \\
\hline 0.22 & 18.29 & 0.55 & $\cdots$ & $\cdots$ & $\cdots$ & $\cdots$ \\
\hline 0.67 & 18.34 & 0.54 & $\ldots$ & $\ldots$ & $\ldots$ & $\ldots$ \\
\hline 1.12 & 18.43 & 0.54 & $\ldots$ & $\ldots$ & $\ldots$ & $\cdots$ \\
\hline 1.58 & 18.54 & 0.53 & $0.106 \pm 0.010$ & $205 \pm 3$ & $-0.02 \pm 0.01$ & $-0.01 \pm 0.01$ \\
\hline 2.04 & 18.66 & 0.53 & 0.103 & 207 & +0.01 & -0.01 \\
\hline 2.51 & 18.79 & 0.53 & $0.096 \pm 0.008$ & 206 & +0.04 & 0.00 \\
\hline 3.00 & 18.94 & 0.53 & 0.091 & $208 \pm 2$ & +0.06 & -0.01 \\
\hline 3.50 & 19.10 & 0.52 & $0.094 \pm 0.005$ & 208 & +0.06 & -0.01 \\
\hline 4.02 & 19.26 & 0.52 & 0.097 & $205 \pm 1$ & +0.06 & -0.01 \\
\hline 4.56 & 19.41 & 0.50 & 0.089 & 204 & +0.04 & 0.00 \\
\hline 5.12 & 19.57 & 0.50 & 0.080 & 207 & +0.04 & 0.00 \\
\hline 5.70 & 19.72 & 0.49 & $0.075 \pm 0.003$ & 210 & +0.05 & +0.02 \\
\hline 6.31 & 19.86 & 0.49 & 0.073 & 210 & +0.07 & +0.02 \\
\hline 6.96 & 20.00 & 0.49 & 0.070 & 212 & +0.10 & +0.01 \\
\hline 7.64 & 20.14 & 0.49 & 0.069 & 212 & +0.13 & +0.05 \\
\hline 8.35 & 20.29 & 0.48 & 0.062 & 216 & +0.15 & +0.02 \\
\hline 9.11 & 20.42 & 0.48 & 0.052 & 214 & +0.14 & +0.03 \\
\hline 9.91 & 20.55 & 0.48 & 0.046 & $212 \pm 2$ & $+0.16 \pm 0.02$ & $+0.02 \pm 0.02$ \\
\hline 10.8 & 20.69 & 0.47 & 0.043 & 218 & +0.15 & +0.04 \\
\hline 11.7 & 20.81 & 0.47 & 0.042 & 221 & +0.18 & +0.10 \\
\hline 12.6 & 20.93 & 0.46 & $0.043 \pm 0.005$ & $226 \pm 3$ & $+0.21 \pm 0.03$ & $+0.14 \pm 0.03$ \\
\hline 13.6 & 21.05 & 0.46 & 0.035 & 228 & +0.21 & +0.07 \\
\hline 14.7 & 21.16 & 0.45 & 0.027 & $231 \pm 4$ & +0.20 & +0.03 \\
\hline 15.9 & 21.26 & 0.45 & 0.032 & 232 & $+0.22 \pm 0.04$ & $-0.06 \pm 0.04$ \\
\hline 17.1 & 21.36 & 0.44 & 0.047 & 233 & +0.22 & 0.00 \\
\hline
\end{tabular}

${ }^{\text {a }}$ One sigma errors $0.01 \mathrm{mag}$ differentially. See text for discussion of zero-point errors. $\mu$ is mag $\operatorname{arcsec}^{-2}$.

${ }^{b}$ One sigma error given applies to the number for which it is given and all subsequent numbers in column until a new error is given.

TABLE 3

\begin{tabular}{|c|c|c|c|c|c|c|}
\hline $\begin{array}{c}\text { Radius } \\
\text { (Arcsec) }\end{array}$ & $\mu_{r}$ & $g-r$ & $\begin{array}{c}\varepsilon \\
(1-b / a)\end{array}$ & $\begin{array}{c}\mathrm{P} . \mathrm{A} . \\
\theta\end{array}$ & $\begin{array}{c}\Delta x^{\prime \prime} \\
(\text { North })\end{array}$ & $\begin{array}{c}\Delta y^{\prime \prime} \\
\text { (East) }\end{array}$ \\
\hline $\begin{array}{l}0.22 \\
0.67 \\
1.12 \\
1.58 \\
2.04 \\
2.51 \\
3.00 \\
3.50 \\
4.02 \\
4.56 \\
5.12 \\
5.70 \\
6.31 \\
6.96 \\
7.64 \\
8.35 \\
9.11 \\
9.91 \\
10.8 \\
11.7 \\
12.6 \\
13.6 \\
14.7 \\
15.9 \\
17.1\end{array}$ & $\begin{array}{l}17.44 \\
17.49 \\
17.58 \\
17.71 \\
17.87 \\
18.06 \\
18.25 \\
18.44 \\
18.63 \\
18.81 \\
18.98 \\
19.15 \\
19.31 \\
19.47 \\
19.61 \\
19.75 \\
19.89 \\
20.02 \\
20.16 \\
20.29 \\
20.41 \\
20.52 \\
20.64 \\
20.78 \\
20.90\end{array}$ & $\begin{array}{l}0.57 \\
0.56 \\
0.56 \\
0.55 \\
0.54 \\
0.54 \\
0.53 \\
0.54 \\
0.53 \\
0.53 \\
0.53 \\
0.52 \\
0.51 \\
0.49 \\
0.49 \\
0.49 \\
0.49 \\
0.48 \\
0.48 \\
0.47 \\
0.47 \\
0.46 \\
0.46 \\
0.46 \\
0.46\end{array}$ & \begin{tabular}{l}
\multicolumn{1}{c}{$\cdots$} \\
$\cdots$ \\
$0.216 \pm 0.008$ \\
$0.214 \pm 0.006$ \\
$0.223 \pm 0.004$ \\
0.252 \\
0.261 \\
0.268 \\
0.278 \\
$0.287 \pm 0.003$ \\
0.295 \\
0.304 \\
0.310 \\
0.317 \\
0.321 \\
0.324 \\
0.326 \\
0.332 \\
0.336 \\
0.349 \\
0.353 \\
0.363 \\
0.358 \\
0.359
\end{tabular} & $\begin{array}{c}\ldots \\
\cdots \\
257.7 \pm 1.1 \\
257.8 \pm 0.7 \\
257.7 \pm 0.5 \\
257.7 \\
257.7 \\
257.9 \\
257.3 \\
257.7 \\
257.9 \\
258.2 \pm 0.4 \\
258.3 \\
258.3 \\
258.3 \\
258.2 \\
258.3 \\
258.2 \\
258.0 \\
258.3 \\
258.4 \\
258.8 \\
258.7 \\
258.8\end{array}$ & $\begin{array}{c}\cdots \\
\cdots \\
0.00 \pm 0.01 \\
0.00 \\
-0.01 \\
0.00 \\
0.00 \\
0.00 \\
0.00 \\
0.00 \\
-0.01 \\
0.01 \\
-0.02 \\
-0.02 \\
-0.03 \\
-0.03 \\
-0.04 \\
-0.03 \\
-0.05 \pm 0.02 \\
-0.07 \\
-0.07 \\
-0.10 \\
-0.09 \pm 0.03 \\
-0.06\end{array}$ & $\begin{array}{l}\cdots \\
\cdots \\
0.00 \pm 0.01 \\
0.00 \\
0.00 \\
-0.01 \\
-0.01 \\
-0.02 \\
-0.01 \\
-0.01 \\
0.00 \\
-0.01 \\
-0.02 \\
-0.03 \\
-0.05 \\
-0.06 \\
-0.08 \\
-0.10 \\
-0.17 \pm 0.02 \\
-0.29 \\
-0.42 \\
-0.46 \\
-0.37 \pm 0.03 \\
-0.42\end{array}$ \\
\hline
\end{tabular}

Notes as for Table 2 . 
TABLE 4

N6251 Photometry (Kitt Peak data)

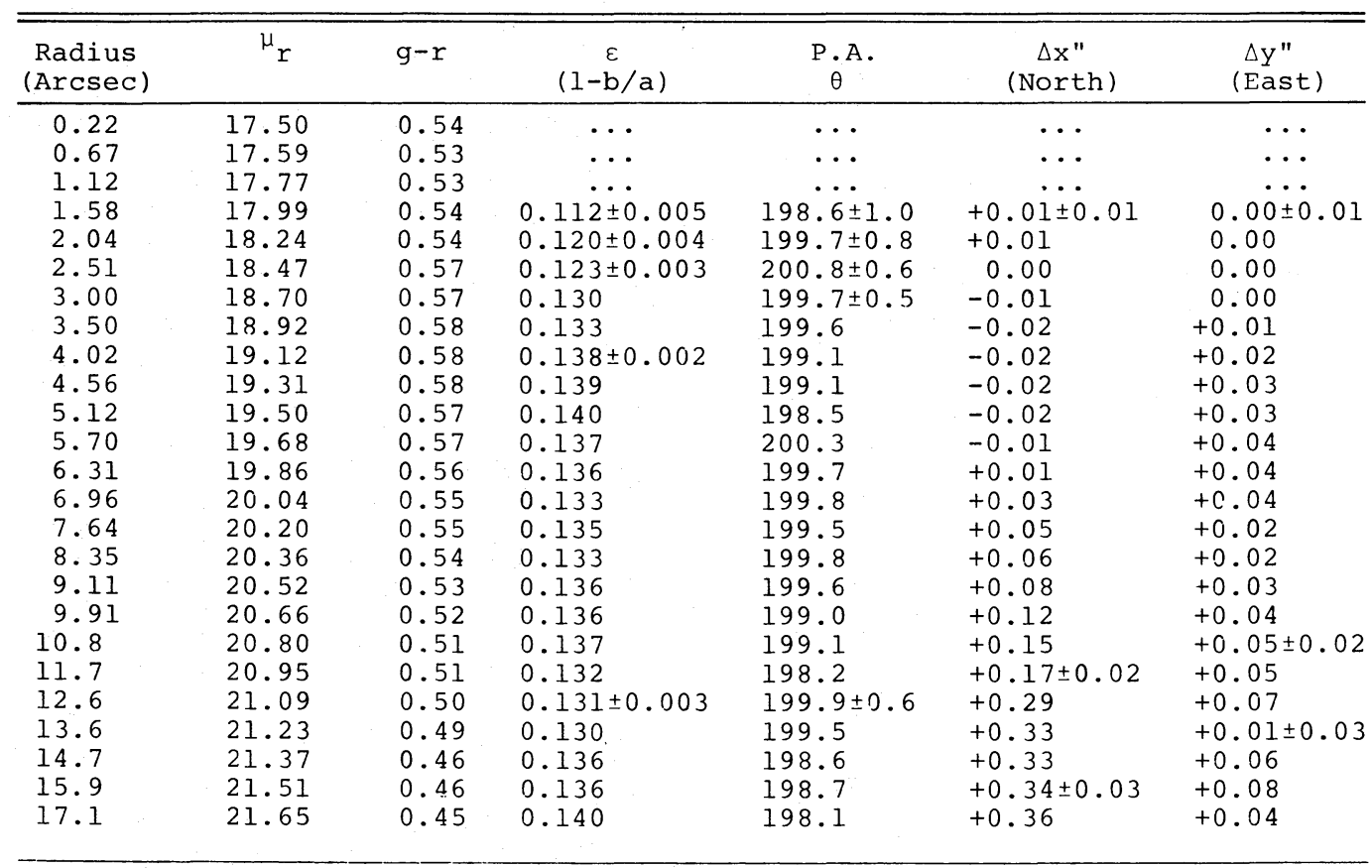

Notes as for Table 2 .

these tables, in the figures, and in the subsequent discussion the term "radius" is used to refer to the radial parameter $s$ defined above in $\S$ III $b$. In Table 5 we give additional data for NGC 6251 based on a 60 second exposure in the $R$ band made at Palomar in exceptionally,good seeing. Spline functions were used to connect these Palomar observations smoothly onto the Kitt Peak data by matching the magnitudes in the region outside $5^{\prime \prime}$ (where seeing does not affect the profile).

The seeing profile for each frame was determined from stars in that frame. We give the Gaussian standard deviation for the inner core of the profile on each frame in Table 1. We note in passing that bright and faint stars on a particular frame had the same

TABLE 5

N6251 PHOTOMETRY ${ }^{\text {a }}$

\begin{tabular}{|c|c|c|c|}
\hline $\begin{array}{l}\text { Radius } \\
(\operatorname{arcsec})\end{array}$ & $\mu_{r}{ }^{b}$ & $\begin{array}{l}\text { Radius } \\
\text { (arcsec) }\end{array}$ & $\mu_{\tau}^{b}$ \\
\hline 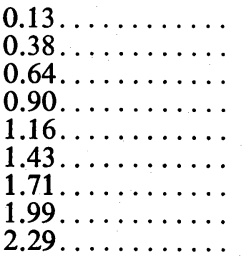 & $\begin{array}{l}16.94 \\
17.05 \\
17.20 \\
17.39 \\
17.59 \\
17.79 \\
18.00 \\
18.18 \\
18.35\end{array}$ & 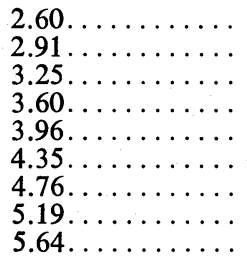 & $\begin{array}{l}18.53 \\
18.67 \\
18.82 \\
18.97 \\
19.12 \\
19.25 \\
19.39 \\
19.52 \\
19.65\end{array}$ \\
\hline
\end{tabular}

a Palomar data.

b One sigma errors $0.01 \mathrm{mag}$ (differentially). $\mu$ is mag $\operatorname{arcsec}^{-2}$.
Gaussian standard deviation for the inner regions. (The outer regions of the faint stars were buried in noise and could not be compared with the wings of the bright stars.) This shows that the CCD did not suffer from charge leakage effects which would broaden the profiles of bright stars relative to those of faint stars.

The galaxy profiles and corresponding star profiles are plotted in Figures 1-3. Aperture photometry of NGC 4874 and NGC 4889 by Gunn and Hoessel (1979, private communication) agrees well with our integrated profiles. The radial gradients in color are quite striking in all three galaxies; they all become increasingly blue at larger radii. NGC 6251 also becomes slightly bluer in the center inside the seeing disk, suggesting the presence of a central nonthermal, blue, point source similar to that observed in M87.

We note from Table 4 that the average position angle of the major axis in NGC 6251 is $199^{\circ} \pm 0.5$. The radio jets observed by Waggett, Warner, and Baldwin (1977) and by Readhead, Cohen, and Blandford (1978) have position angles of $296^{\circ} .5 \pm 0.5$ and $300.5 \pm 2^{\circ}$, respectively. The optical minor axis is $289^{\circ} \pm 0.5$ which seems significantly different, but by only a few degrees, from the direction of the radio jets.

\section{PROFILE FITTING}

\section{a) King Models}

We first attempted to fit the measured light profiles of each of the three galaxies with a King (1966) model using nonlinear least squares to find the parameters. These models contain as a parameter the "cutoff" energy $\epsilon_{T}$ defined such that the stellar distribution 


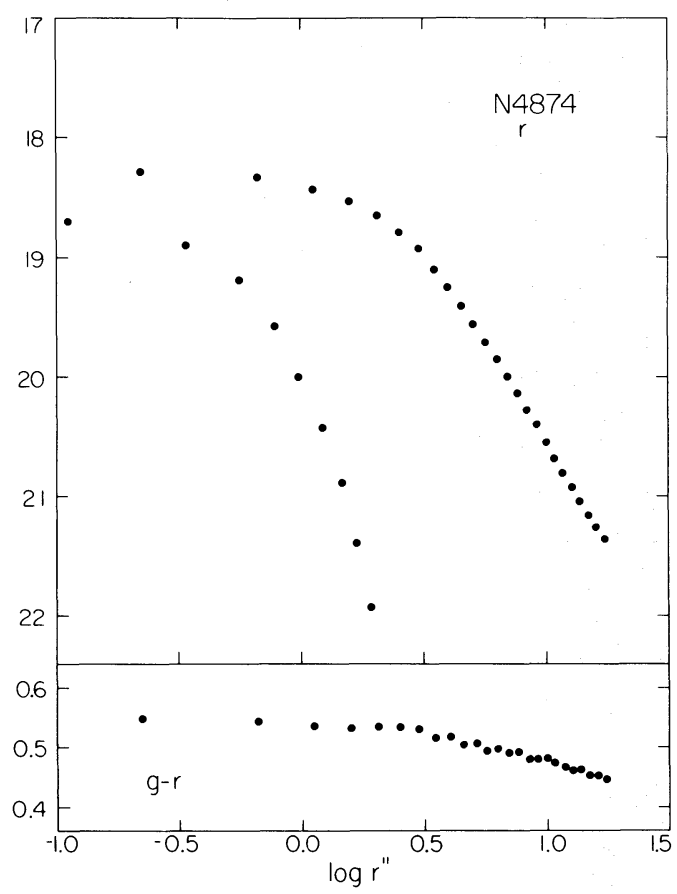

FIG. 1.-Photometry of N4874. The ordinate of the upper graph is $\mu_{r}$ (mag $\operatorname{arcsec}^{-2}$ ), and we depict both the galaxy (outer points) and a star (inner points) from the same CCD frame. Below we give the color $g-r$. The radius scale $r^{\prime \prime}$ is in $\operatorname{arcsec}$.

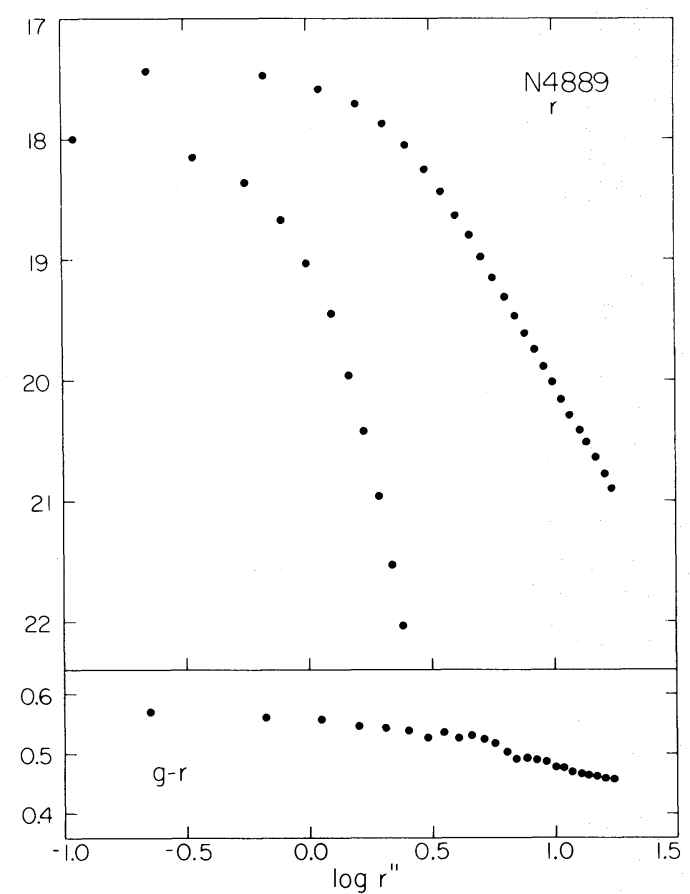

FIG. 2.-Photometry of N4889. Other notes as for Fig. 1.

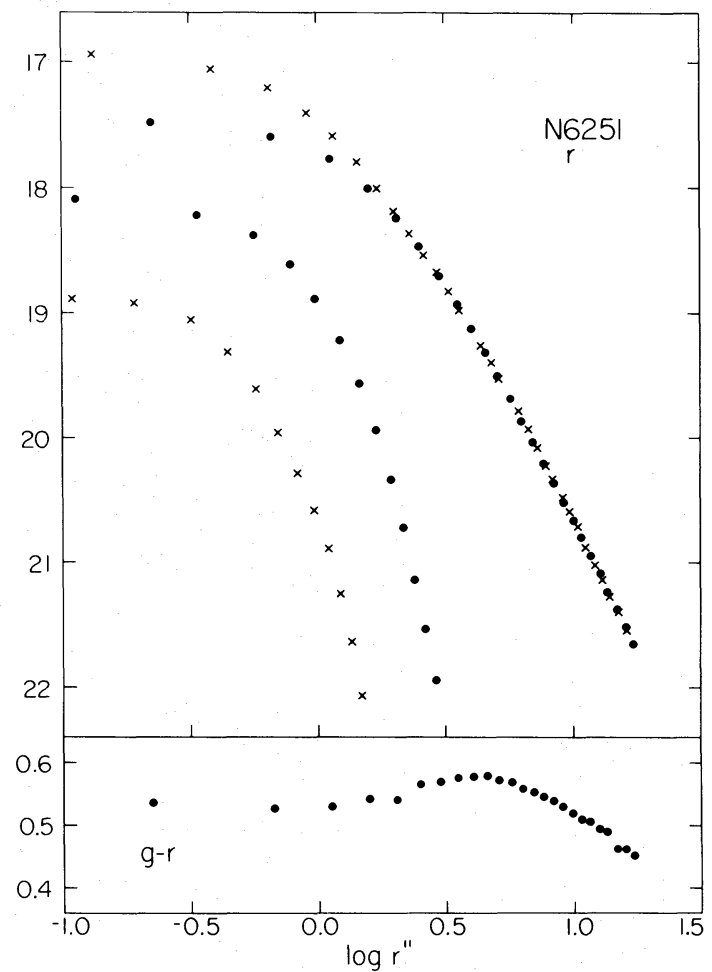

Fig. 3.-Photometry of N6251. Other notes as for Fig. 1. Kitt Peak data (galaxy and star) are given by the filled circles, and Palomar data are given by the crosses (crosses are not plotted when superposed on a filled circle).

function $f(E) \propto \exp \left(-E / \sigma_{v}{ }^{2}\right)-\exp \left(-E_{T} / \sigma_{v}{ }^{2}\right)$, where $\epsilon_{T}=E_{T} / \sigma_{v}^{2}$. Since we are concerned only with the nuclei of the galaxies, $\epsilon_{T}$ is poorly determined by our measurements. Consequently, for each profile we chose an approximate value of $\epsilon_{T}$ and then adjusted the core radius $r_{c}$ and central luminosity so as to obtain the best fit to the observations. Each model was convolved with the appropriate seeing profile (including both Gaussian core and wings to $5^{\prime \prime}$ radius) as the fit progressed. In the absence of nonspherically symmetric models we have fitted the observed elliptical profiles to King's spherically symmetric models. Only for NGC 4889 [with ellipticity $(a-b) / a \sim 0.3$ ] should this be a questionable procedure.

The parameters determined are given in Table 6 for NGC 4874 and NGC 4889. These two galaxies are fitted well by the King models (see Fig. 4) and display large, well-resolved core radii as is characteristic of supergiant elliptical galaxies. On the other hand, NGC 6251 fares less well. We have attempted to fit a composite profile of Palomar and Kitt Peak data for this galaxy with a King model, but the best obtainable fit is very poor (see Fig. 4). As in the case of M87 (Young et al. 1978), the profile turns into the core too gently to be fitted with isothermal spheres.

\section{b) Fits to NGC 6251}

First we compare the light profile of NGC 6251 with the profile of M87 which was obtained in the $V$-band 

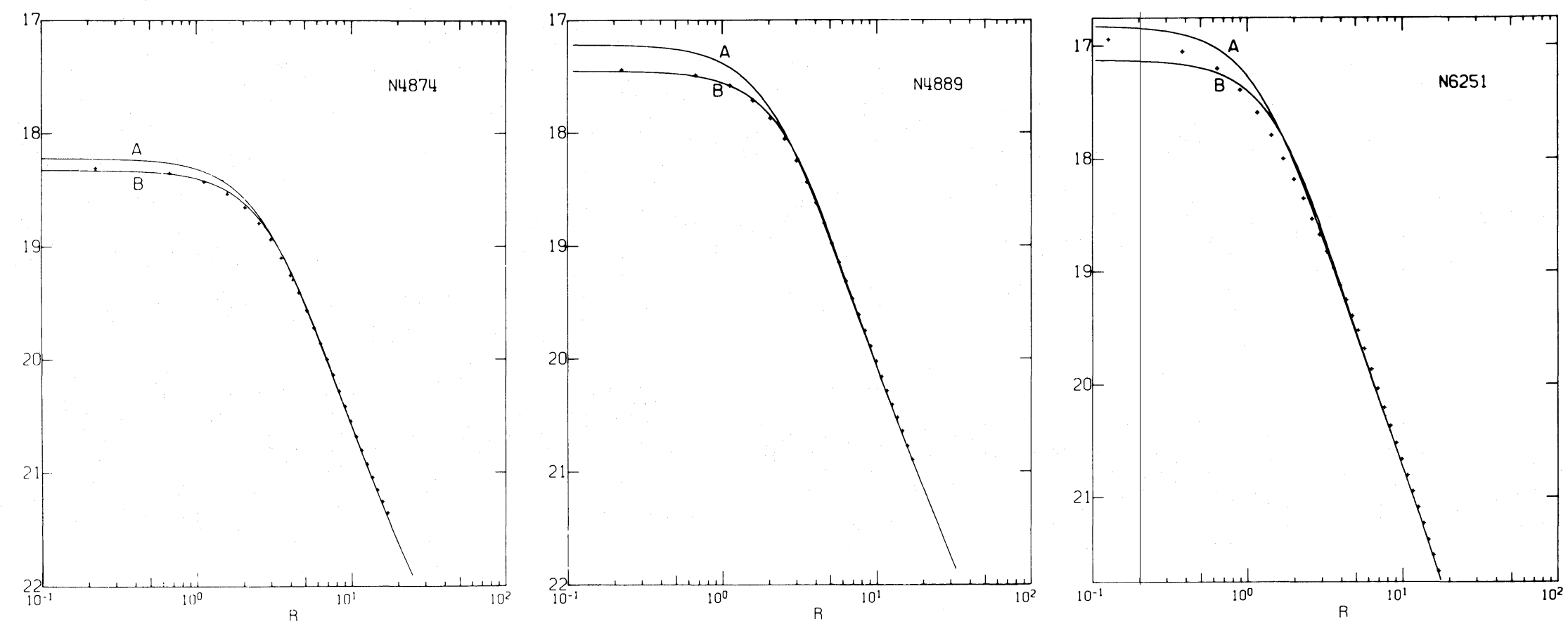

FIG. 4-King models fitted to the galaxy profiles. The ordinate is $\mu_{r}\left(\mathrm{mag} \operatorname{arcsec}^{-2}\right)$ and the abscissa $R$ is in arcsec. For each of the three galaxies the data points are given by pluses. Curve $\mathrm{A}$ is the best fitting King model, and curve B is the same King model convolved with the seeing disk (it is curve $\mathrm{B}$ which attempts to fit the data). 


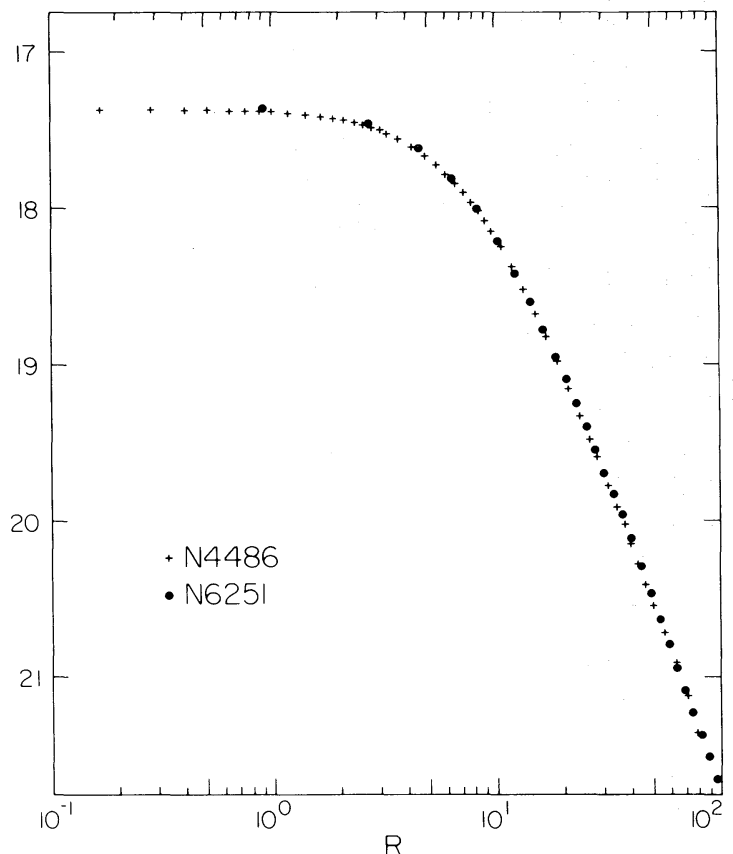

Fig. 5.-Comparison between photometric profiles of $\mathrm{N} 4486$ and N6251. The ordinate is $\mu \mathrm{mag} \operatorname{arcsec}^{-2}$ (in the $V$-band for N4486; $r$ for N6251), and the abscissa $R$ is in arcsec. The N4486 data (pluses) were convolved with $\sigma=3^{\prime \prime}$ seeing and reside at their actual place in the $(\mu, R)$ plane. The N6251 data (circles) have been slid in $\mu$ and $R$ to fit the N4486 data (the seeing disk for the N6251 data, also slid in $R$, defines the seeing $\sigma$ with which the N4486 data should be convolved).

by Young et al. (1978). Such a comparison is made in Figure 5 where, to allow for the difference in distance between the two galaxies, the measured profile of M87 has been convolved with a Gaussian seeing disk with $\sigma=3$. 0 . The two galaxies are seen to be remarkably similar.

With this result in mind, we next attempted to fit NGC 6251 with a King model plus a point luminosity source. The slight blue trend of the galaxy color toward the center (see Fig. 3) leads to the suspicion that a point, nonthermal source may be present. However, as Young et al. (1978) found with NGC 4486, this model is also not very successful (see Fig. 6). Residuals as large as 0.15 mag appear near the core regions; these are unacceptably large in view of the high accuracy of the CCD photometry.
It may be worthwhile to consider the possibility that dust absorption produces the observed departure of the profile of NGC 6251 from the isothermal model. Inspection of Figures 3 and 4 show that the greatest luminosity discrepancy is at $r=2^{\prime \prime}-3^{\prime \prime}$ where the color $(g-r)$ is reddest. We believe that the observed effect is not due to dust absorption for the following reasons.

1. The galaxy NGC 6251 is definitely an elliptical (rather than an S0): it does not show the disk component characteristic of S0's.

2. The isophotes show the luminosity defect to be circularly symmetric in the coordinate $s$ (and, furthermore, to be smooth rather than patchy). Late S0 galaxies tilted to the line of sight generally show asymmetric absorption, often patchy.

3. The normal reddening relation is $A_{r}=2.1 E(g-r)$. The observed fluctuations in NGC 6251 would require $A_{r}=5 E(g-r)$; i.e., a much larger than normal amount of neutral extinction would be needed.

We are finally driven to using models with a central point mass ("black hole models") which fit the data reasonably well (see Fig. 6). Since the observed light profile is similar to that of M87 we expect, and obtain, a similar dimensionless black hole mass, namely, $\mu_{H}=0.6$. The derived luminosity of the central point source in NGC 6251 is rather brighter than that in M87 in absolute magnitude, although this parameter is not very well determined for NGC 6251 because of the poor resolution of the observations relative to NGC 4486.

Finally, we have fitted black hole models to NGC 4874 and NGC 4889 in order to determine the maximum allowable black hole mass. The upper limits, given in Table 6 , are $\mu_{H} \sim 0.1$, some 6 times smaller than detected in NGC 6251 .

\section{DISCUSSION}

Our main results are:

1. NGC 4874 and NGC 4889 have luminosity profiles well represented by King models.

2. NGC 6251 has a luminosity profile which is not fitted by a King model with or without an additional central, point luminosity source. Its profile can, however, be fitted with a "black hole" model and is very similar to that of M87 when the latter is convolved with a large seeing disk.

TABLE 6

Profile Fitting Parameters

\begin{tabular}{|c|c|c|c|c|c|c|c|}
\hline Galaxy & $\begin{array}{l}\text { Filter } \\
\text { Band }\end{array}$ & $\begin{array}{l}\text { Cutoff } \\
\text { Energy }\end{array}$ & $\begin{array}{l}\text { Core } \\
\text { Radius } \\
\text { (arcsec) }\end{array}$ & $\begin{array}{l}\text { Central a } \\
\text { Luminosity }\end{array}$ & $\begin{array}{l}\text { Point } \\
\text { Luminosity }\end{array}$ & $\begin{array}{l}\text { Black } \\
\text { Hole } \\
\text { Mass }^{c}\end{array}$ & $\begin{array}{c}c z \\
\left(\mathrm{~km} \mathrm{~s}^{-1}\right)\end{array}$ \\
\hline $\begin{array}{l}\mathrm{N} 4874 \ldots \ldots \ldots \\
\mathrm{N} 4889 \ldots \ldots \ldots \\
\mathrm{N} 6251 \ldots \ldots \ldots \ldots \\
\mathrm{N} 4486^{\mathrm{b}} \ldots \ldots \ldots \ldots\end{array}$ & $\begin{array}{c}r \\
r \\
r / R \\
V\end{array}$ & $\begin{array}{r}11 \\
10 \\
9 \\
9\end{array}$ & $\begin{array}{l}3.31 \pm 0.06 \\
2.48 \pm 0.04 \\
1.42 \pm 0.08 \\
9.6 \pm 0.5\end{array}$ & $\begin{array}{l}18.23 \pm 0.01 \\
17.20 \pm 0.01 \\
16.86 \pm 0.04 \\
17.20 \pm 0.02\end{array}$ & $\begin{array}{c}\cdots \\
17.8 \stackrel{ }{\cdots} 0.2 \\
16.69 \pm 0.05\end{array}$ & $\begin{array}{l}<0.15 \\
<0.1 \\
\\
0.6 \pm 0.1 \\
\\
0.6 \pm 0.1\end{array}$ & $\begin{array}{l}7176 \\
6467 \\
7104 \\
1287\end{array}$ \\
\hline
\end{tabular}

${ }^{a}$ Measured in mag $\operatorname{arcsec}^{-2}$.

${ }^{b}$ From Young et al. 1978.

${ }^{\mathrm{C}}$ Expressed in terms of $\mu_{\mathrm{H}}$, i.e., as a fraction of the core mass. 

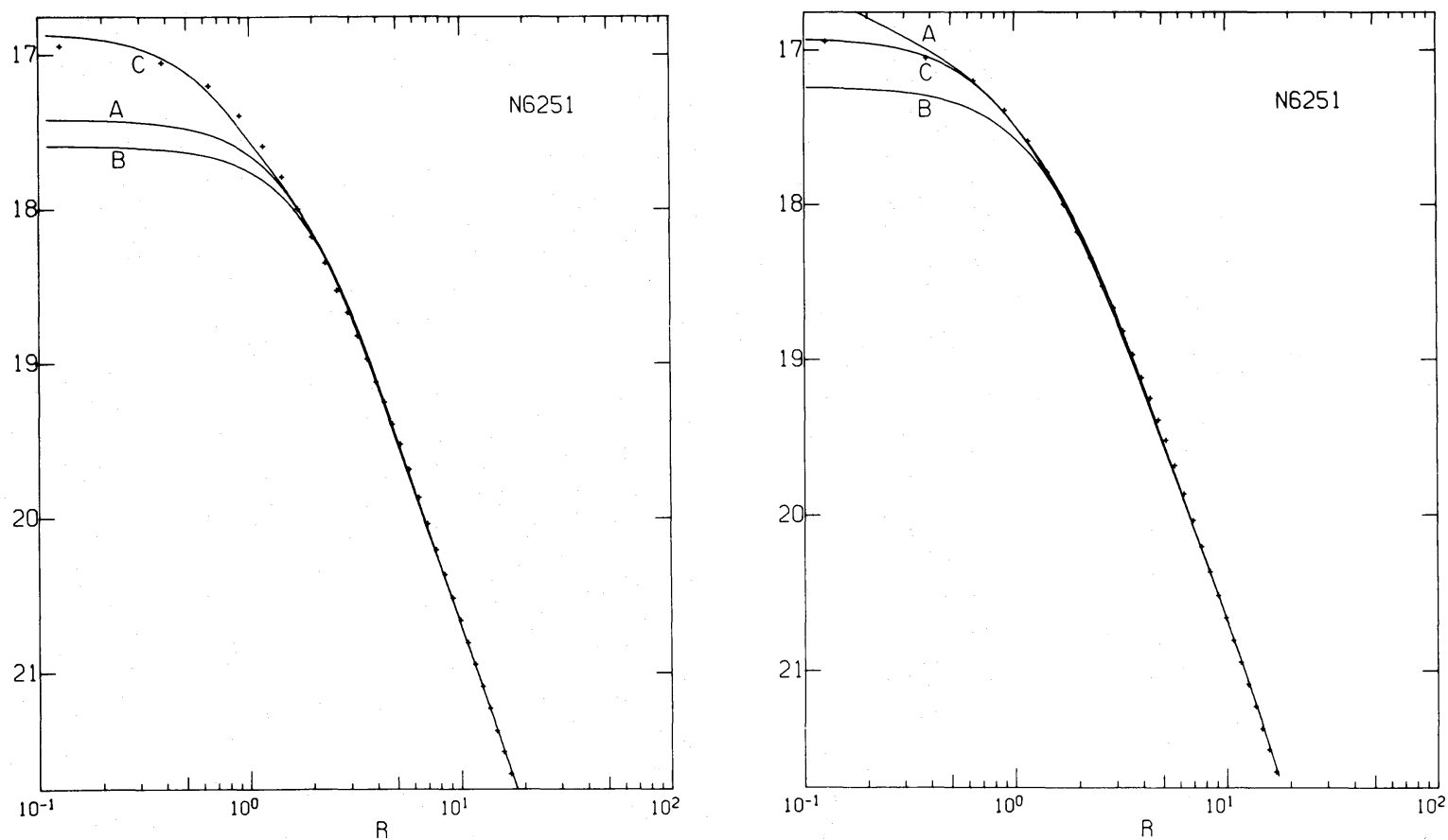

Fig. 6.-Further fits to the N6251 profile. The ordinate is $\mu_{r}\left(\mathrm{mag} \mathrm{arcsec}^{-2}\right)$, and the abscissa $R$ is in arcsec. The left-hand plot shows the best fitting King model plus added central point luminosity source. The right-hand plot shows the best fitting black hole model plus point luminosity source. In both cases curve $A$ is the underlying model, curve $B$ is that model convolved with the seeing disk, and curve $\mathbf{C}$ the convolved model plus a point luminosity source (also convolved).

The mass derived from the black hole model for NGC 6251 is

$$
M_{H}=2.4 \times 10^{9} h_{60}^{-1}\left(\sigma_{v} / 250 \mathrm{~km} \mathrm{~s}^{-1}\right)^{2} M_{\odot},
$$

where $h_{60}=. H_{0} / 60 \mathrm{~km} \mathrm{~s}^{-1} \mathrm{Mpc}^{-1}$ and $\sigma_{v}$ is the core velocity dispersion of NGC 6251 which has not yet been measured. However, an empirical relationship between absolute magnitude $M_{B(0)}$ and $\sigma_{v}$ has been found for elliptical galaxies by Faber and Jackson (1976) and confirmed by Sargent et al. (1977). These last authors derived the relation

$$
\begin{aligned}
\log \sigma_{v} & =0.215-0.100 M_{B(0)} \\
& \pm 0.466 \pm 0.023
\end{aligned}
$$

from their data. If we insert the value $M_{B(0)}=-21.3$ for NGC 6251 into equation (9), we find $\sigma_{v}=221 \mathrm{~km}$ $\mathrm{s}^{-1}$. Consequently, we expect that the value of $M_{H}$ finally estimated for NGC 6251 will turn out to be very close to that found for M87, namely, about $2 \times 10^{9} M_{\odot}$. It would clearly be of interest to obtain dynamical data on NGC 6251. The angular resolution of $1^{\prime \prime}$ obtainable from the ground would compare favorably in linear resolution with the $5^{\prime \prime}$ obtained on M87 (Sargent et al. 1978).

We are still far from being able to prove that the central mass concentrations found in M87 and now in NGC 6251 are really black holes, rather than concentrations of stars whose value of $M / L$ is much higher than that of the stellar population in the main bodies of these galaxies. Nevertheless, it seems remarkable that these anomalous central light distributions have so far only been discovered in two radio galaxies whose nonthermal activity is very similar.

Two of us (P. J Y. and W. L. W. S.) are extremely grateful to the Director and staff of the Kitt Peak National Observatory for the opportunity to participate in the trials of the JPL CCD camera. We are especially grateful to Dr. Roger Lynds of Kitt Peak and to Greg Cozza of JPL for their considerable help with the observations. This work was supported by NSF grants AST 76-81089 to W. L. W. S. and AST 77-04182 to the Carnegie Institution of Washington.

\section{REFERENCES}

Arp, H. C. 1967, Ap. Letters, 1, 1.

de Vaucouleurs, G., and Nieto, J. L. 1979, Ap. J., $230,697$.

Faber, S. M., and Jackson, R. E. 1976, Ap. J., 204, 668

King, I. R. 1966, A.J., 71, 64.

Readhead, A. C. S., Cohen, M. H., and Blandford, R. J. 1978, Nature, 272, 131 .

Readhead, A. C. S., Sargent, W. L. W., and de Bruyn, G. 1979, $A p . J .$, in press.
Sargent, W. L. W., Schechter, P. L., Boksenberg, A., and Shortridge, K. 1977, Ap. J., 212, 326.

Sargent, W. L. W., Young, P. J, Boksenberg, A., Shortridge, K., Lynds, C. R., and Hartwick, F. D. A. 1978, Ap. J., 221, 731.

Thuan, T. X., and Gunn, J. E. 1976, Pub. A.S.P., 88, 543.

Waggett, P. C., Warner, P. J., and Baldwin, J. E. 1977, M.N.R.A.S., 181, 465 . 
Willson, M. A. G. 1970, M.N.R.A.S., 151, 1.

Young, P. J, Westphal, J. A., Kristian, J., Wilson, C. P., and Landauer, F. P. 1978, Ap.J., 221, 721.
Zwicky, F., and Herzog, E. 1968, Catalogue of Galaxies and Clusters of Galaxies, Vol. 4 (Pasadena: California Institute of Technology).

Jerome Kristian: Hale Observatories, 813 Santa Barbara Street, Pasadena, CA 91106

Wallace L. W. Sargent and Peter J Young: Department of Astronomy 105-24, California Institute of Technology, Pasadena, CA 91125

James E. WestPhal: Division of Geological and Planetary Sciences, California Institute of Technology, Pasadena, CA 91125 